\title{
A Scalable Architecture for Supporting Interactive Games on the Internet
}

\author{
Wen tongCAI P ercival XAVIER Stephen J. TURNER Bu-Sung LEE \\ $\mathrm{P}$ arallel \&Distributed Processing Laboratory \\ School of Computer Engineering \\ Nany ang Technological University \\ Singapore 639798
}

\begin{abstract}
This paper presents a scalable archite ctur efor supporting large-sale inter active Internet games. In order to support a large number of particip antsand to divide the workload, the virtual world is divided into partitions. Each partition is then assigned to a server. A client (i.e., a player or a particip ant) will join a server ac ording to the position of the avatar it controls. Compared to a centr alizel archite cture, this distributd client-server archite cture is mor e sablab. In addition, compared to a fully distribute d,peer-to-p eerarchite $c$ ture, it also provides a means for dete cting cheating in distribute dgames. Sinc einteractions and accounting information must be forwarded directly to one of the servers for qualification and verific ation, cheating amongst distribute dplayers of the game will be minimized. To supprt secur al communication for interactions and accounting information as well as to speedup periodic update messages (e.g., position updates), a hybrid communication scheme using both TCP and IP multicast is used betwe enclients and the asso ciated server. The communication among servers is enabled by the $R$ un-Time Infrastructure ( $R$ TI) services. The High Level Archite cture (HLA) Data Distribution Management (DDM) is employed to limit the amount of communication betwe enthe servers. In addition, the Ownership Management (OM) is also employed to implement the ne edfor transferring the avatars betwe en servers. In this $p$ ap er, the design detail of the anhite cture will be presente $d$. An experimental interactive Internet game realize dusing the archite cture will $b$ e also describ $\boldsymbol{d}$ in the paper.
\end{abstract}

Keywords: Large-scale Interactiv eGames, Scalability, Distributed Client-Serv er Arditecture, High Level Architecture (HLA), Run-Time Infrastructure (RTI),
Ownership and Data Distribution Management.

\section{Introduction}

Interactive multi-user Internet games have their origins from programs sharing a common heritage known variously as MUGs (Multi-User Games), MUDs (MultiUser Dungeons or Multi-User Dimensions) and MUAs (Multi-User Adven tures). They hav e gained significant popularity due to their en tertainmert value. Along with the improvements to computer graphics, audio and real-time processing, multi-user games have also improved in terms of visual interactivit y. T o accommodate the great demand for reality and in teractivity, information critical for rendering of remote entities must be issued as frequently as possible. This simple scheme is however inadmissible because of the following tw o factors [9]:

- The ever increasing requirement for state updates of remote entities will ov erload the simulation engine; and

- Net w orkatency and limited bandwidth will put an upper bound to the rate at which entities can exc hange information with eadh other.

These tw o factors lead to the issue of software architectural scalability. A scalable softw are arc hitecture can be defined as a general framework that supports a virtual environment with an increasingly larger $n$ umber of concurrent dynamic entities and/or players without fundamental modifications to that architecture. The design of a softw are arc hitecture mst take the abo ve tw o factors into account because faster computers and netw orks alone will not satisfy the requirements for increasing the number of participants in a virtual environment over time. 
Most of the currently available, in teractiv emultiuser Internet games are based on a centralize d architectur e(i.e., client-server arc hitecture), where all the clien ts (i.e., pla yers) are connected to a centralized server. The communication betw een the clierts will go through the server and the sever maintains a consistent game view of all the clients. So, the major problem of this architecture is that the server will become the bottlenec $\mathrm{k}$ in terms of both comminication and computation, thus limiting the scalability. A logical solution to this problem is to have a fully-distributed architecture, where each client computes its own view of the game state and communicates with other clients without the intervention of a server. An example application using the fully-distributed architecture is the MiMaze which is a distributed multiplay er in teractiv e game deakeped using IP multicast [3]. In addition, the High Level Architecture (HLA) [2, 4] also provides a framework for constructing such fully-distributed, interactiv e, nultiuser In ternet games.

Another important area of concern in interactiv e multi-user Internet games is securit y. One major aspect of securit $y$ is the prevention of cheating among clien ts participating in the game. In a centralized architecture, information reaches its destination through the server. Therefore, a consistent state of scoring and accounting can be easily maintained and game companies, for example, can charge pla yers based on the duration of their participation. How ever,in a fully-distributed architecture, since interactions and accounting information are not qualified and verified by a server, cheating amongst distributed pla yersof the game is possible. Detection of cheating in a fullydistributed architecture is also more difficult than that in a centralized architecture.

Hence, the objective of this paper is to develop a distribute d client-server axhite ctur for in teractive Internet games, which combines the advantages of both centralized and fully-distributed architectures. The virtual world is divided into partitions. Each partition is then assigned to a server. A clien $t$ will join a server according to the position of the avatar ${ }^{1}$ it con trols. An overview of the architecture will be presented in Section 2.

To preserve interactivit y of the game, a fast response from the server is required. The strategy applied is to incorporate a hybrid communication mechanism depending on the nature of the information that is passed betw een clierts and the server. In Section 3, a detailed

\footnotetext{
${ }^{1}$ We use the word "client" or "player" to refer to a physical participan t in the interactive Internet game and the word "avatar" to refer to the graphical embodiment representing the participan $t$ in the virtual en vironmen
}

account of the design strategy of the front-end clientserver communication will be discussed.

The communication among servers is enabled by the services provided by the Run-Time Infrastructure (R TI) of the HLA. Our work is in-line with the areas in volving distributed virtual en vironmerts [10], netw ork edvirtual environments [9] and distributed simulations based on the HLA [6]. A common technique used by these applications to limit the amount of data pac kets being transmitted in the network is data filtering (or interest management). HLA Data Distribution Management (DDM) services are employ ed to limit the amount of communication betw een the serers. The issues concerning the design of the back-end servers will be studied in Section 4 .

In Section 5, an experimental in teractiv eIn ternet game realized using the architecture will be also described. The conclusions and the description of future work will be given in Section 6 .

\section{An Overview of the Distributed Client-Server Architecture}

F ully-distributed and cen tralized arc hitectures can be combined to form a distributed client-serv er arc hitecture where there are multiple servers pro viding services to the clients. The distributed client-serv er arc hitecture retains the advantage of the simple centralized (clien t-server) architecture. In addition, by sharing the load of computation and communication amongst multiple serv ers, more plyers would be able to participate in the same virtual environment. There are two principal approaches to divide the work amongst servers in a distributed client-serv er arc hitecture [1:

- Virtual World Subdivision: The virtual w orldis partitioned in to logical groups and eac $\mathrm{h}$ group is assigned to a server. Clients connect to the server according to the group to which its a vatar belongs.

- Participant Sub division Clients are grouped and assigned to a server according to the physical distance between the clien $t$ and the serv er. Clients connect to the server according to the geographical area in which they are located.

In the virtual world subdivision approach, a server only maintains a part of the entire virtual $\mathrm{w}$ orld. But, a clien t may need to migrate to a different server if it changes its logical group. In the participant subdivision approach, a client will connect to the same server throughout the game. But, each server may need to maintain a cop y of the en tire virtual world. An example application using participant subdivision can be 
found in [7]. In this paper, we adopt the virtual world subdivision approach.

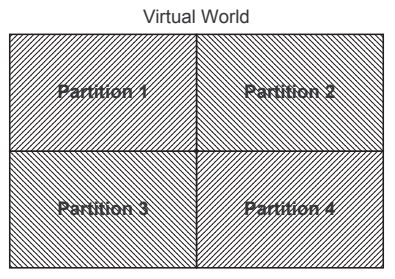

(a)

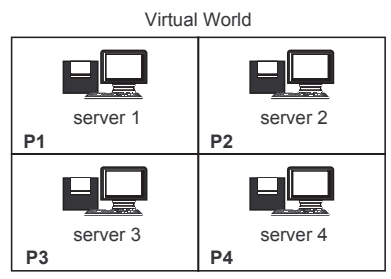

(b)

\section{Figure 1. Spatial Partition of Virtual World}

An optimum choice for distributing load amongst the servers may depend on the application, netw ork topology and other design decisions. In [8], it suggests three possibilities for partitioning a virtual world:

- Sp atial Partitioning This is based on partitioning the virtual world into areas which can be processed in parallel and independently. Therefore, the players in the same part of the virtual world can interact with each other through the same server.

- Tempral Partitioning: Entities that require the same rate of update are grouped together. Groups requiring a higher update rate can then have a larger share of the total netw ork bandwidth.

- F unctional Partitioning Entities are grouped according to functional classes (e.g., a battalion in $\mathrm{w}$ ar-gaming). Those in the same functional class can then communicate with each other frequently through a multicast group.

In this paper, the spatial partitioning approach is adopted. As shown in Figure 1(a), the virtual world is spatially divided into partitions. A server is then assigned to each partition, managing a group of players (clien ts) who ha veavatars in the partition (Figure 1(b)). Thus, each serv er is responsible for managing only a portion of participating players in the entire virtual environment.

Figure 2 sho ws the overall communication infrastructure of the distributed clien t-serv erarchitecture. In this infrastructure, the servers comply with the HLA rules and communicate with each other through the R TI. The clierts connected to the same server are represen ted $\mathrm{b}$ y the serer as a federate in the federation. Communication and interaction between clien ts and the server is via socket connections.

Figure 3 sho ws a more detailed design of the distributed clien t-serv erarc hitecture. It consists of tw o main parts: the front-end client-server and the distributed back-end servers. Each serv er consists of three modules: server back-end, message queue and server front-end. The main role of the server back-end module is to process interactions, accounting information and update messages of the participating pla yers and to $\mathrm{k}$ eep state information of all the avatars in the partition. Server back-end modules are in fact the federates participating in the federation. They together with the R TI form the distributed bakk-end servers. The main aim of using an HLA-based implementation is to enhance interoperability and scalability. When an avatar moves from one partition to another, the HLA Ownership Management (OM) services are used to migrate the corresponding avatar betw eenthe tw oservers involv ed. In order to reduce the amount of data transmitted among the servers, the HLA DDM services are employed so that a server only subscribes to the state updates of remote avatars near the edge of its partition. The functionality of the distributed bac k-end servers will be further explained in Section 4 .

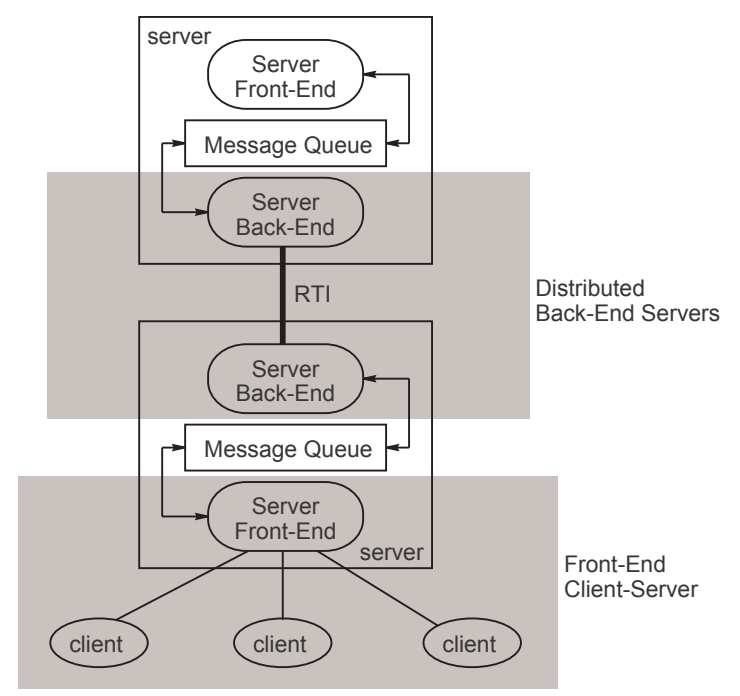

\section{Figure 3. Design of Distributed Client-Server Architecture}

The front-end client-serv er structure consists of the server fron t-end module and the associated clients. The main task of the server fron t-end module is to handle the arriv al of joining clien ts and to provide a mechanism for the server to communicate with the clients. The server bac k-end and froth-end modules communicate with each other through a message queue. The development of the fron t-end cliert-serv er structure will 


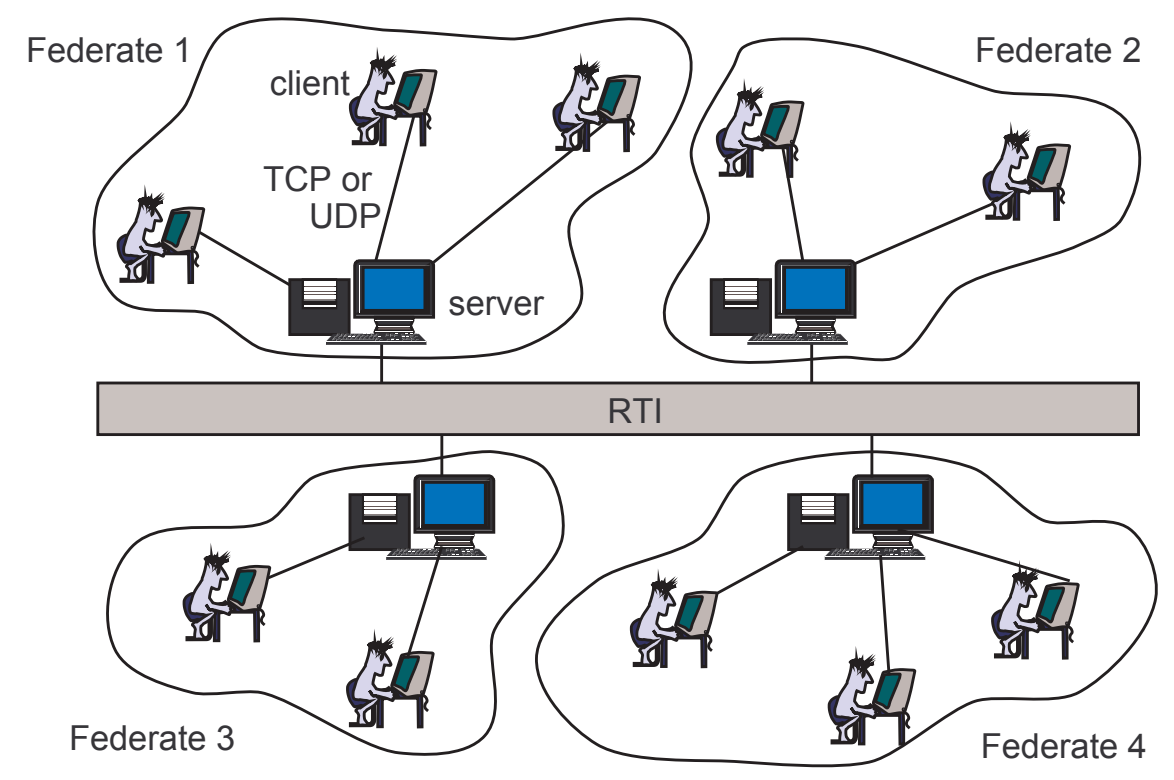

Figure 2. Overview of Distributed Client-Server Architecture

be further explained in the next section.

\section{Front-end Client-Server Structure}

A communication architecture is required for information exchange betw een clients and the server. There are three types of message-passing in a front-end clientserv er structure: position updates, interactions and accounting information. Clients need to issue position updates which will be sent to the server and will also be propagated to other clien ts in the structure. The serv er will use these updates to maintain the state of the virtual environment so as to verify clien ts' interactions. Clients must also forward interactions (e.g., attac king actions), when attempting to in teract with the virtual environment, to the server. The server will qualify the interactions and sends results back to the clien $t$. The accounting information is sent betw een the serv er and clients for session management and scoring. Table 3 giv es a summary of the relativ efrequency of these three types of client-serv er message-passing.

$\mathrm{T}$ o ensure the securiy of data, a connection-oriented approach is required for handling interactions and accounting information. Thus, a TCP connection is established betw een each clien $t$ and the server. IP multicast relies on a connectionless, datagram-based approach to route pac ketsbetween netw orken tities. Since no acknowledgment pack et from the receiving en-

\begin{tabular}{c|c} 
Type & R elative Frequency \\
\hline P osition Updates & High \\
In teractions & Medium \\
Accounting & Low
\end{tabular}

Table 1. Classification of Client-Server Communication

tity is required, information passing betw een netw ork en tities can tale place at a faster rate than TCP. With this advantage, IP multicast is deployed to handle position updates of clients because these updates are required to be transmitted at a higher frequency as compared to interactions and accounting information. A major problem with IP multicast is the possibility of lost packets. This issue is how ev er ignored because the occasional loss of position updates does not severely affect the simulation of remote avatars.

Figure 4 shows a logical view of the communication scheme used in the front-end clien t-serverstructure. The server fron t-endmodule is multithreaded. There is a thread for each TCP connection and a separate thread for handling position updates. These threads act as proxies betw een the distributed badk-end servers and the clients, relaying messages in both directions. The message queue is used to buffer the messages be- 


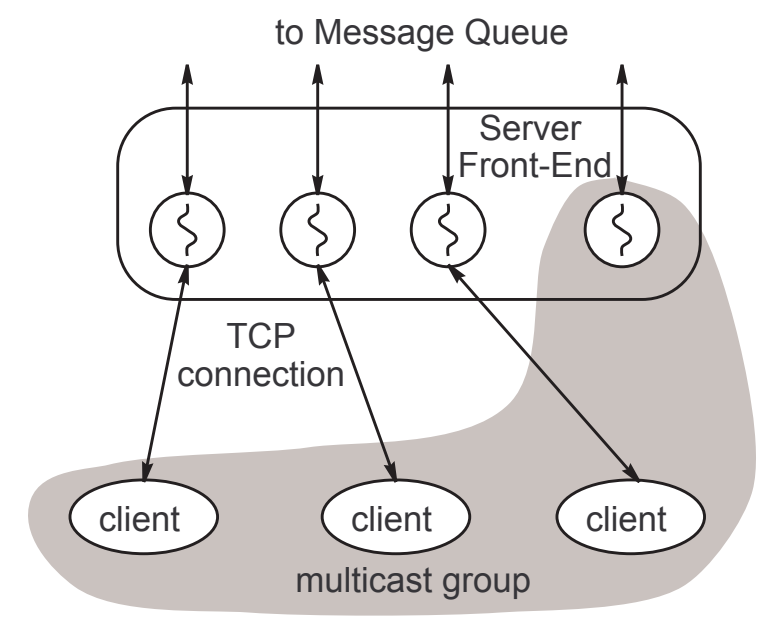

Figure 4. Communication Architecture for Front-end Client-Server

tw een the front-end client and the distributed back-end serv ers. The shaded area in Figure 4 represents a multicast group which includes all the clients connected to the same server and the thread responsible for position updates. As explained above, this multicast group is used for transmitting position updates.

In summary, to support secured communication for interactions and accounting information as well as to speedup periodic update messages (e.g., position updates), a hybrid communication arc hitecture using both TCP and IP multicast is used in the fron t-end clien t-serv er structure.

\section{Distributed Back-end Servers}

Distributed back-end servers consist of server backend modules, each of which is built as a federate and is responsible for maintaining state updates of all avatars it owns and handling interactions and accounting information. As discussed in Section 2, the spatial partitioning sc heme is adopted in the design of the distributed clien t-serv er arc hitectureThe selection is based on the assumption that at any time, the participating avatars are likely to be uniformly distributed in the virtual world since they are able to move around in the virtual world autonomously. One problem in the spatial partitioning scheme is the migration of an avatar from one server ${ }^{2}$ to another when it changes its partition.

\footnotetext{
${ }^{2}$ Server in this section actually refers to the server back-end module of the server.
}

This is illustrated in Figure 5 where the virtual world is spatially divided into four partitions. When an avatar changes from partition $P_{1}$ to partition $P_{3}$, the ownership of the avatar should be transferred from server 1 to server 3 . In addition, the clien $\mathrm{t}$ which controls the avatar should also be reconnected to server 3 . The HLA's Ownership Management (OM) services are used to solv e this problem.

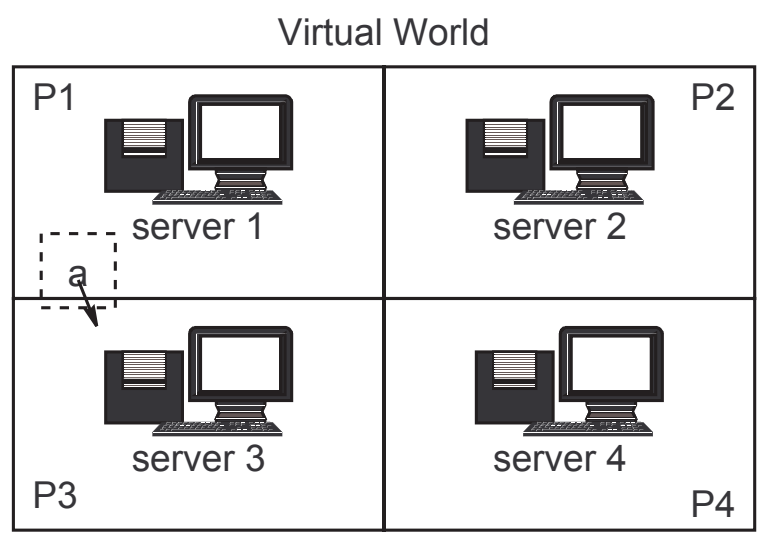

Figure 5. Migration of Client and Avatar

In order to ensure a seamless virtual en vironment, an a vatar located at the edge of the partition mst be able to "see" the close-b yavatars belonging to other partitions so that they can interact with each other. So, another problem is how to maintain a seamless virtual en vironment without resulting in too much inter-serv er traffic. To solve this problem, the HLA's Data Distribution Management (DDM) services are employed.

\subsection{Transferring Ownership of Avatars}

The HLA's OM services are deployed to transfer ownership of avatars when they cross the boundaries of the partition to which they currently belong. In order to enforce a scheme for ownership management of a vatars, each server must be able to perform a real-time query of each avatar's position to determine whether an avatar has moved out of the server's partition. The interaction diagram in Figure 6 shows how the transfer of a vatar o wnership is drieved. Dotted lines represent communications in the distributed back-end severs and solid lines represent communications in the front-end clien t-serv er structure.

The ownership of each avatar is divested based on the ownership push scheme. Ownership push suggests that a federate that owns update responsibility 


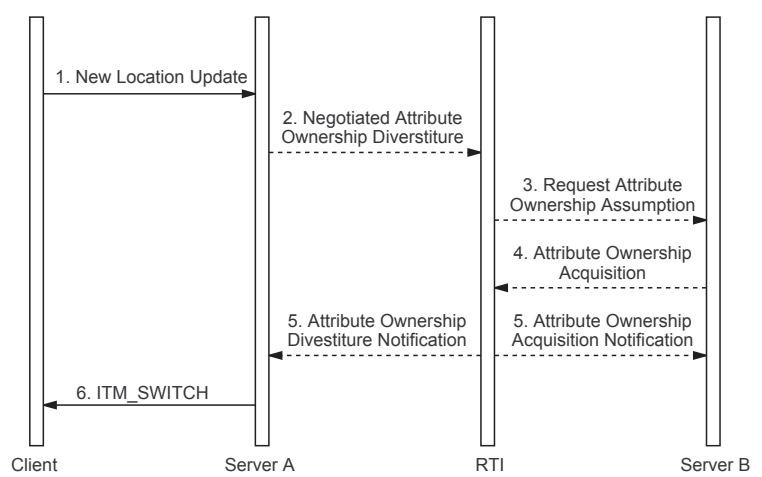

Figure 6. Protocol for Transferring Ownership of Avatar

of and/or the privilege to delete instance attributes wishes to transfer ownership of the attributes to another federate. The ownership may be surrendered unconditionally or by ne gotiation Unconditional push releases a federate from attribute update and/or deletion responsibility without any commitment from other federates to assume these responsibilities. Negotiated push is a formal exchange where a federate retains responsibility until a new owner is identified and a formal exc hange process is completed. The negotiated push scheme is adopted in our design.

As sho wn in Figure 6, a server wishing to let go the responsibilities calls the $\mathrm{R}$ TIanbassador method negotiatedAttributeOwnershipDivestiture(). As only one server may acquire the ownership of an avatar, the div esting server will specify which server shall be the new owner of its div ested avatar. The specification is made through the tag data when negotiatedAttributeOwnershipDivestiture() is called. Given that the other servers are capable of updating any or all of the attributes being giv en aw ay,they are therefore, notified via their $\mathrm{F}$ ederateAmbassador method requestAttributeOwnershipAssumption(). A server wishing to acquire one or more of the offered attributes indicates its interest using the method attributeOwnershipAcquisition(). If any server is found to assume the responsibilities being given aw ay, the server that initiated the push receives attributeOwnershipDivestitureNotification(). The server gaining the responsibilities is informed with attributeOwnershipAcquisitionNotification(). Once the transaction for ownership management is done, the server which originally owned the avatar instance will send a message ITM_SWITCH to the clien t to inform it of the need to join another multicast group. It is one type of the interaction messages sent betw een the server and clients [11]. The IP address of the new server and the multicast group address are pro vided in the ITM_SWITCH message.

\subsection{Provision of State Updates of Edge Avatars}

The HLA's DDM services are used to provide state updates of the avatars near the edge of a partition. Serv ers exhange avatar state information by updating and reflecting object attribute values. And they obtain only relevan $t$ state updates from neighboring partitions by defining update and subscription regions.

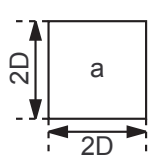

(a)

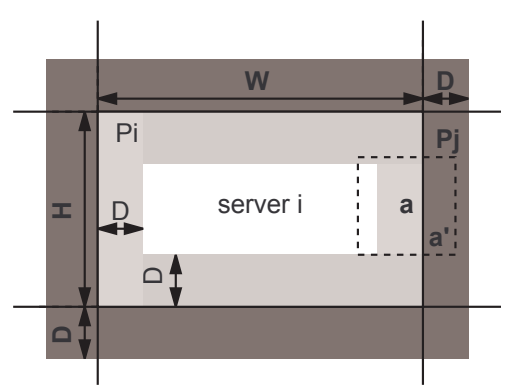

(b)

\section{Figure 7. Update and Subscription Regions}

The extent to which an avatar can see another is defined by the avatar's view radius $D$. Factors affecting this value depend on the virtual world's en vironmental conditions (e.g., fog and obstructions) as w ell as the speed of the avatar. Figure 7(a) shows the dimensions of the part of the virtual world displayed for each pla yer. Figure 7(b) sho ws a server's update and subscription regions. The update region of a server is the en tire partition assigned to the server. In this case, the update region of server $i$ is the partition $P_{i}$ itself. The subscription region of a server is the region surrounding its partition. In Figure 7(b), the subscription region of server $i$ is represented by the darkly-shaded area. DDM services associateRegionForUpdates() and subscribeObjectClassAttributesWithRegion() are used. A server only updates the state of an avatar, by updateAttributeValues(), if it is in the edge of the partition (for server $i$ in Figure 7(b), it is represented by the lightly-shaded area.

F or example, as shown in Figure 7(b), when avatar $a^{\prime}$ moves into the darkly-shaded anea partition $P_{j}$, server $j$ will update its position. The update region of $a^{\prime}$ is the partition $P_{j}$ itself and the subscription region 
of the server $i$ is the darkly-shaded area. So, there is an overlap betw een update and subscription region. The position update of $a^{\prime}$ will be sent to serv eri. Therefore, avatar $a$ in partition $P_{i}$ will be able to see avatar $a^{\prime}$ in partition $P_{j}$ in its display ed virtual world.

Obviously, the advan tage of using the DDM services is the reduction on inter-serv er communication. Now, eac $h$ server only receives the updates of relevant avatars from other servers. The other advantage of the above definition of update and subscription regions is that they are constant and need not be dynamically recreated. Thus, the overhead on calculating the overlap betw een regions is minimized.

\section{An Experimental Internet Game}

An experimental in teractive Internet game has been constructed using the distributed clien t-serv erarchitecture. The game scenario is reminiscent of "Pacman" in which each player has a 3D representation of his/her view of the game (with respect to Figure 7(a)). The game involv esa group of avatars that na vigate through a virtual 3D maze with the objective of finding as many items as possible. Items are generated by the virtual environment dynamically at random positions. Avatars may group into teams (according to their colors). Avatars may also attack one another to "steal" items. How ev er, an datar that attacks its o wn team member will result in a penalty. Figure 8 shows the view of a player. In this case, there are tw oremote avatars and one static item in the view. The selfrepresentation is ignored in the view since each player is assumed to view the virtual world from his/her own angle.

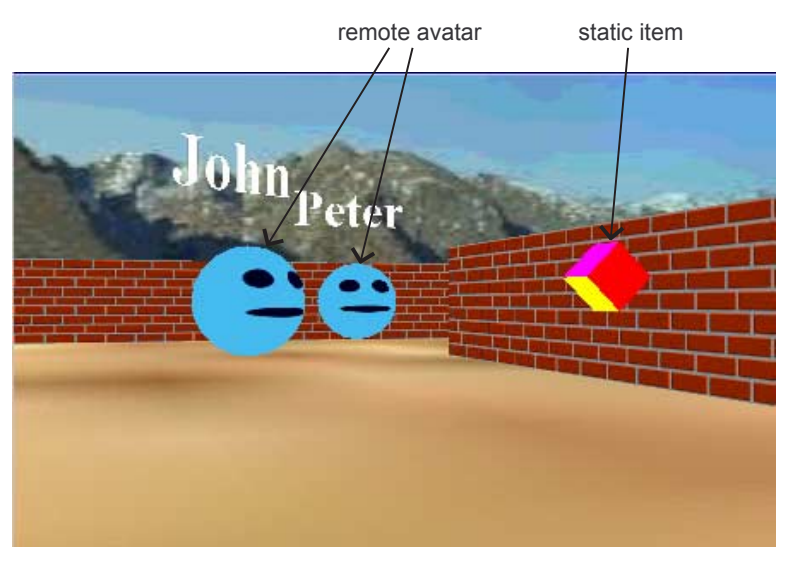

Figure 8. View of a Player
Preliminary performance analysis has been conducted using M/M/1 queue to compare centralized and distributed clien t-serv erarchitectures. Currently, experiments are being conducted to collect results from the experimental Internet game for further performance study.

\section{Conclusions and Future Work}

The development of the distributed client-server architecture is divided into t wo stages. The first stage is concerned with the development of a front-end clientserver structure that enables client-server interaction. Both IP multicast and TCP based communication are deploy ed to relay different classes of information. The advantage of multicast lies with its abilit y to route pac kets at a fast rate.As such, it is employed to transmit position updates betw een participating clients. TCP, on the other hand, is employ ed to establish a secure communications channel betw een the cliert and its server to rela y accouning information and interactions.

The second stage of dev elopmert is in volv edwith the construction of the distributed back-end servers. The main purpose of employing a distributed architecture is to divide the workload of managing avatars among multiple of servers. The scheme employed for scalability is based on spatial partitioning. In this scheme, there is a need to transfer the management of avatars when they migrate from the scope of interest of their current server. To maintain a seamless virtual $\mathrm{w}$ orld, a vatars located at the edge of a partition need to "see" avatars belonging to other partitions. The $\mathrm{R}$ TIis used for the communication in the distributed back-end servers, and the HLA services are employed to address the above two issues. The HLA Ownership Management services are used to transfer the ownership of clien $t$ instances. $T$ o enable the edges of partitions to be visible to neighboring partitions, the DDM management services were employ ed to create update and subscription regions.

F uture w ork on thisgame architecture will in volve an improv ement on the distribution of clients. In the current scheme, the distribution of workload for managing clien tinteractions is static and is based on the assumption that clients will be uniformly spread out in the virtual world. How eer, if clients are not uniformly distributed, the workload will become unbalanced. Another problem with the current design is that a client needs to migrate to a different server when it changes its partition. In case an area close to a boundary crossing becomes a hot spot, the overhead of clien ts constantly switching betw een serers might be heavy. T o 
overcome this problem, a hysteresis approach can be adopted in the definition of update and subscription regions. T ofurther improve the load balance and to minimize client migration ov erhead, a hybrid approach using both virtual $\mathrm{w}$ orldand participant subdivision will also be investigated.

\section{References}

[1] T. K. Capin, et. al. A vatarsin networked virtual envir onmentsWiley \& Sons Inc, West Susses, 1999.

[2] Judith Dahmann, Frederic kKuhl and Ric hard Weatherly. Standards for simulation: As simple as possible but not simpler - The High Level Architecture for simulation. Simulation, 71:6, pp.378-387, 1998.

[3] C. Diot and L. Gautier. A distributed architecture for multiplayer in teractiv eapplications on the internet. IEEE Network, pp.6-15, July/August 1999.

[4] DMSO. Department of Defense High Level Architecture specification. V ersion 1.3 DMSO, 1999 (available at http://hla.dmos.mil).

[5] C. Heistad and S. Pietrowicz. Y ou-build-it virtual reality. NCSA Ja va3D Grp., Univ. of Illinois Urbana-Campaign, $1999 \quad$ (a vailable at http://www.ncsa.uiuc.edu/SDG/Softw are /Java3D/).

[6] F. Kuhl, R. Weatherly and J. Dahmann. Creating computer simulation systems - A nintr duction to the High L evel A rchite ctur.e Prentice Hall PTR, NJ, 1999.

[7] T. C. Lu, C. N. Lee and W. Y. Hsia. Supporting large-scale distributed simulation using HLA. $A C M$ Transactionson Modelling and Computer Simulation, Vol.10, No.3, pp.268-294, July 2000.

[8] Macedonia et. al. Npsnet: A multi-player 3D virtual environment over the Internet. in Proc. Symposium on Interactiv e 3D Graphics, ACM, pp. 9394, 1995.

[9] Sandeep Singhal and Michael Zyda. Networked virtual environments: Design and implementation. Addison-Wesley, 1999.

[10] Martin R. Stytz. Distributed virtual en vironments. IEEE Computer Graphics and Applications, pp. 19-31, May 1996.

[11] reference removed for double-blind review process 